\title{
Damit Implantate nicht krank werden Professionelle Zahnreinigung zur Vorbeugung und Behandlung von Periimplantitis
}

reddot design award winner 2008

Die moderne Implantattechnik gilt im Allgemeinen als eine sehr sichere Methode, um verloren gegangene, natürliche Zähne zu ersetzen. Gerade ihre hohe Erfolgsrate macht diese Art von Zahnersatz für Patienten attraktiv. Dennoch ziehen Implantate im Laufe der Zeit immer häufiger Folgeprobleme mit sich. Oft ist es schwierig, die Patienten nach dem Implantateingriff zu regelmäßigen Kontrolluntersuchungen zu motivieren. Gerade die Nachsorge ist aber überaus wichtig. Wird sie vernachlässigt, sammeln sich an den Implantaten wie auch bei natürlichen Zähnen Beläge an, die aus Speiseresten, Bakterien und Speichelbestandteilen bestehen. Werden diese nicht entfernt, entsteht zunächst eine periimplantäre Mukositis, eine Entzündung der Schleimhaut über dem Implantat. Diese heilt ohne Weiteres wieder aus, sobald die verursachenden Beläge beseitigt sind.

Bleiben die Beläge allerdings bestehen, kann es zu einer Periimplantitis kommen, das heißt einer entzündlichen Erkrankung des Zahnfleisches und des Knochens rund um das Implantat. Sie geht mit einem Knochenverlust einher. Im schlimmsten Fall wird so viel Knochen abgebaut, dass es zur Lockerung und schlussendlich zum Verlust des Im-

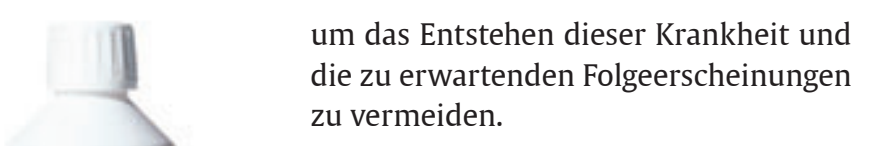

Sub- und supragingivale Beläge sicher entfernen

Mit der neuen Behandlungsform AirFlow handy Perio kann der Zahnarzt eine Periimplantitis bei Patienten effektiv vorbeugen und bestehende Zahnfleischentzündungen wirkungsvoll behandeln. Nach Aussage des Herstellers ist das Gerät das erste und einzige tragbare Periogerät, das eine sichere Entfernung der subgingivalen Beläge ermöglicht. Das Sprayhandstück hat eine speziell designte biokompatible Einwegdüse, mit der es gelingt, den kompletten Biofilm sowie andere Beläge bis zum Taschenboden zu entfernen. Die in einem Gemisch aus Pulver, Luft und Wasser applizierte biokinetische Energie reduziert zudem die Bakteriendichte nachhaltig, strafft das Zahnfleisch und verringert die Taschentiefe.

Für Praktiker, die es darüber hinaus schätzen, mehrere Funktionen in einem Gerät zu nutzen, soll die unabhängige Prophylaxe-Einheit Air-Flow Master Abhilfe schaffen. Das Gerät kümmert sich nicht nur um die subgingivale Prophylaxe, sondern entfernt auch die Zahnoberflächen von Plaque und harten Belägen.

Sowohl der Air-Flow Master als auch das Air-Flow handy Perio sollen gemeinsam mit dem feinen $25 \mu \mathrm{m}$, glycinbasierten, nicht abrasiven Perio-Pulver eine wirkungsvolle Reinigung der Implantate sowie der natürlichen Zähne ermöglichen.

Sylvia Bösch, Köln die Wahrscheinlichkeit, dass sich eine Knochenentzündung entwickelt. Je nach Situation wird mit dem Patienten die nächste Sitzung individuell festgelegt, 